\title{
Intermediate risk of multidrug-resistant organisms in patients who admitted intensive care unit with healthcare-associated pneumonia
}

Hongyeul Lee, Ji Young Park, Taehoon Lee, Yeon Joo Lee, Hyo-Jeong Lim, Jong Sun Park, Ho Il Yoon, Jae-Ho Lee, Choon-Taek Lee, and Young-Jae Cho

Division of Pulmonary and Critical Care Medicine, Department of Internal Medicine, Seoul National University Bundang Hospital, Seongnam, Korea

Received: April 17, 2015

Revised : July 2, 2015

Accepted: September 8, 2015

\section{Correspondence to}

Young-Jae Cho, M.D.

Division of Pulmonary and

Critical Care Medicine,

Department of Internal

Medicine, Seoul National

University Bundang Hospital, 82

Gumi-ro 173beon-gil,

Bundang-gu, Seongnam 13620,

Korea

Tel: $+82-31-787-7058$

Fax: +82-31-787-4051

E-mail: lungdrcho@snubh.org
Background/Aims: Healthcare-associated pneumonia (HCAP) was proposed as a new pneumonia category in 2005 , and treatment recommendations include broad-spectrum antibiotics directed at multidrug-resistant (MDR) pathogens. However, this concept continues to be controversial, and microbiological data are lacking for HCAP patients in the intensive care unit (ICU). This study was conducted to determine the rate and type of antibiotic-resistant organisms and the clinical outcomes in patients with HCAP in the ICU, compared to patients with community-acquired pneumonia (CAP) or hospital-acquired pneumonia (HAP). Methods: We conducted a retrospective cohort analysis of patients with pneumonia $(\mathrm{n}=195)$ who admitted to medical ICU in tertiary teaching hospital from March 2011 to February 2013. Clinical characteristics, microbiological distributions, treatment outcomes, and prognosis of $\operatorname{HCAP}(n=74)$ were compared to those of CAP $(n=75)$ and $\operatorname{HAP}(n=46)$.

Results: MDR pathogens were significantly higher in HCAP patients (39.1\%) than in CAP $(13.5 \%)$ and lower than in HAP $(79.3 \%, p<0.001)$. The initial use of inappropriate antibiotic treatment occurred more frequently in the HCAP (32.6\%) and HAP $(51.7 \%)$ groups than in the CAP group (11.8\%, $p=0.006)$. There were no differences in clinical outcomes. The significant prognostic factors were pneumonia severity and treatment response.

Conclusions: MDR pathogens were isolated in HCAP patients requiring ICU admission at intermediate rates between those of CAP and HAP.

Keywords: Community-acquired pneumonia; Healthcare-associated pneumonia; Hospital-acquired pneumonia; Intensive care units; Multidrug-resistant pathogens

\section{INTRODUCTION}

Pneumonia is one of the most common infectious diseases requiring admission to the intensive care unit (ICU) for medical treatment. With an aging population, the number of patients who receive care at facilities other than hospitals, such as long-term healthcare facilities, assisted-living environments, or rehabilitation facilities are increasing. Therefore, the traditional classifications for pneumonia based on the patient's location before admission such as community-acquired pneumonia (CAP) or hospital-acquired pneumonia (HAP) needed to be updated $[1,2]$, consequently, a new term, healthcareassociated pneumonia (HCAP) was introduced by the 
Infectious Diseases Society of America (IDSA) and the American Thoracic Society (ATS) in 2005 [3].

Patients who develop HCAP are more similar to hospitalised patients than to independently living community-based patients, in that they have a greater burden of comorbidities, including cancer, chronic kidney disease, heart disease, chronic obstructive lung disease, immunosuppression, dementia, and impaired mobility $[1,3,4]$. These diverse spectra of HCAP patients may result in varied epidemiology and patient-specific risks for antibiotic-resistant pathogens [5-7].

To address this, the IDSA/ATS guidelines recommend broad empirical antibiotic therapy followed by culture-guided de-escalation for patients with HCAP [3]. However, despite an excellent negative predictive value (96\%), the IDSA/ATS criteria have a low positive predictive value $(18 \%)$ for differentiating a true infection or colonization with multidrug-resistant (MDR) bacteria in patients with HCAP who admitted to the ICU [8]. Therefore, the adherence to these guidelines is not required in all cases and is able to result in the overuse of antibiotics [9]. Moreover, the current approach to HCAP treatment is also in the need of revision [10-12].

Herein, we tried to determine the differences in the presence of antibiotic-resistant organisms and clinical outcomes in HCAP patients who need ICU care, compared with CAP and HAP patients

\section{METHODS}

\section{Study subjects and design}

From March 2011 to February 2013, we conducted a prospective cohort in a 16-bed medical ICU and a retrospective analysis of patients who required an ICU admission for pneumonia. A clinical diagnosis of pneumonia required the presence of new radiographic infiltrates and at least 2 of the following clinical criteria: fever (> $\left.38^{\circ} \mathrm{C}\right)$ or hypothermia $\left(\leq 35^{\circ} \mathrm{C}\right)$, new cough with or without sputum production, pleuritic chest pain, dyspnoea, or altered breath sounds on auscultation. We excluded patients with a documented do-not resuscitate order. The decision of admission to ICU was done in the case of who was required for close monitoring with septic shock under vasopressor or acute respiratory failure requiring intubation and mechanical ventilation [3].
We define HAP as pneumonia that developed after being hospitalised for $>48$ to 72 hours and HCAP as pneumonia that also met at least 1 of the following criteria: (1) recent history of hospitalisation for $\geq 2$ days within 90 days of the infection; (2) residence in a nursing home or long-term care facility; (3) recent intravenous antibiotic therapy, chemotherapy, or wound care within 30 days prior to the current infection; or (4) attendance at a haemodialysis clinic [3]. Patients with pneumonia who did not meet any of the criteria for HCAP or HAP were identified as having CAP. We compared clinical characteristics, pneumonia severity, the distribution of pathogens, and outcomes between the three groups (CAP, HCAP, and HAP). If patients admitted to the ICU for pneumonia $\geq 2$ times during one hospital admission, only the first event of pneumonia was included. The Institutional Review Board Committee of Seoul National University Bundang Hospital waived the informed consent in this study (No. B-1105/127-001).

\section{Microbiological studies}

At the day of ICU admission, microbiological studies were conducted using two sets of blood culture samples, gram staining and culture using the transendotracheal aspirate or sputum from patients without intubation, and when available, a bronchoscopic lower respiratory tract culture that was obtained by bronchoscopy at the bedside of ICU. Obtained samples were cultured in a semi-quantitative manner.

An etiological diagnosis was made when a respiratory pathogen was isolated from a sterile specimen, a pneumococcal antigen was detected in urine, the antibody titers for an atypical pathogen increased 4 -fold or converted to positive, or a predominant micro-organism was isolated from adequate sputa $(>25$ neutrophils and $<10$ squamous epithelial cells per low-power field) or bronchial washing or alveolar lavage fluids with compatible gram staining results. Methicillin-resistant Staphylococcus aureus (MRSA), drug-resistant strains of Pseudomonas aeruginosa, Acinetobacter species, Stenotrophomonas maltophilia, and extended-spectrum $\beta$-lactamase (ESBL)-producing Enterobacteriaceae were considered to be MDR pathogens, as previously reported [13].

\section{Antibiotic therapy}

Empirical antibiotic therapy was defined as the use of 
any antibiotics for $>48$ hours during the first 3 days of admission. Broad-spectrum antibiotics were defined as the use of any antibiotics that included anti-pseudomonal $\beta$-lactamase, vancomycin, or carbapenem.

Antibiotics therapy was initiated after at least blood culture samples were done because of severe condition requiring admission to ICU in basic accordance with the ATS/IDSA guideline [3]. However, the detailed antibiotic regimen complied with the attending physician's choice taking into consideration patient's risk factors and the severity of the disease. The appropriateness of antibiotic therapy was analysed for all cases with an etiological diagnosis according to susceptibility test criteria for lower respiratory tract pathogens. Antibiotic therapy was classified as being inappropriate if the initially prescribed antibiotics were not directed at the identified pathogens, and treatment failure was defined as death during the initial treatment or poor treatment response. Poor treatment response defined as a change in the empirical antibiotics from the initial agents within the 7 th day of the ICU admission.

\section{Statistical analysis}

To compare the differences between the groups, Fisher exact tests were used for categorical variables, and the two-tailed $t$ test, analysis of variance, or Mann-Whitney test was used for continuous variables, as appropriate. Statistical significance was established at a two-tailed $p=$ 0.05. All analysis was conducted using SPSS version 18.0 (SPSS Inc., Chicago, IL, USA).

\section{RESULTS}

\section{Baseline characteristics}

During the study period, 195 patients that required ICU care for pneumonia were eligible for the study: 75 with CAP (38.1\%), 74 with HCAP (37.6\%), and 46 with HAP (24.4\%) (Table 1). Distribution of HCAP were described in Table 2, Supplementary Table 1, and HAP in Supplementary Table 2. Patients with HCAP were significantly more likely to have comorbidities, particularly cerebrovascular disease ( $55.4 \%$ vs. $30.7 \%, p=0.009$ ) and chronic kidney disease ( $16.2 \%$ vs. $1.3 \%, p=0.002)$, than CAP patients. Leukopenia was also significantly more common in patients with HCAP than in those with CAP $(23.0 \%$ vs. $5.3 \%, p=0.005)$. There were no significant differences in pneumonia severity measured using the confusion, urea, respiratory rate, age $\geq 65$ (CURB-65) criteria $(\geq 3$ ) and pneumonia severity index (PSI; high-risk class). Disease severity according to the Acute Physiology and Chronic Health Evaluation II (APACHE II) and Sequential Organ Failure Assessment scores was similar across the three groups.

\section{Pathogen distribution}

All of the patients had results of the gram staining and cultures of their blood and sputum. The number of bronchoscopic lower respiratory tract specimens was 44 in HCAP patients (61.1\%), 50 in CAP patients (66.7\%), and 23 in HAP patients $(60.9 \%, p=0.730)$. Bacterial pathogens were identified in 46 HCAP patients $(62.2 \%), 37$ CAP patients (46.3\%), and 29 HAP patients (63.0\%). Table 3 lists the frequency of each of the etiologic micro-organismsfor each group. The most common pathogens were methicillin-susceptible Staphylococcus aureus (MSSA) (29.7\%), Streptococcus pneumoniae (13.5\%), and P. aeruginosa (10.8\%) in the CAP patients; Klebsiella pneumonia (45.6\%), MRSA (19.6\%), and Escherichia coli (15.3\%) in the HCAP patients; and MRSA (44.8\%), P. aeruginosa (24.1\%), and K. pneumonia (24.1\%) in the HAP patients.

In all three groups, $S$. aureus was the most common gram-positive pathogen. Of the $S$. aureus pathogens, MSSA was detected significantly more often in the CAP group than in the HCAP and HAP groups $(p=0.001)$. MRSA was detected at comparable rates in the CAP and HCAP groups and significantly more often in the HAP group $(p=0.002)$. Of the gram-negative pathogens, HCAP and HAP patients had significantly higher rates of ESBL-producing Enterobacteriaceae than the CAP patients $(p=0.015)$.

The prevalence of MDR pathogens in the HCAP group (39.1\%) was significantly higher than in the CAP group ( $p$ $<0.005)$ and lower than that in the HAP group $(p=0.001)$ (Fig. 1). Inappropriate initial antibiotic treatment was administered significantly less often in the CAP group $(p=0.034)$ than in the HCAP and HAP groups $(p=0.146)$.

\section{Antimicrobial treatment and clinical outcomes}

In all three groups, the majority of the patients received combination antibiotic therapy as the initial treatment (CAP 86.7\%, HCAP 78.4\%, and HAP 71.7\%) (Table 4). 
Table 1. Baselines characteristics of the study groups

\begin{tabular}{|c|c|c|c|c|}
\hline Characteristic & $\mathrm{CAP}(\mathrm{n}=75)$ & $\operatorname{HCAP}(\mathrm{n}=74)$ & $\operatorname{HAP}(\mathrm{n}=46)$ & $p$ value \\
\hline Age, yr & $72(19-90)$ & $73(32-99)$ & $75(46-91)$ & 0.409 \\
\hline Male sex & $59(78.7)$ & $56(75 \cdot 7)$ & $38(79.2)$ & 0.872 \\
\hline \multicolumn{5}{|l|}{ Comorbidities } \\
\hline Chronic lung disease $^{\mathrm{a}}$ & $29(38.7)$ & $17(23.0)$ & $15(31.3)$ & 0.117 \\
\hline Chronic heart disease & $29(38.7)$ & $32(43.2)$ & $24(50.0)$ & 0.350 \\
\hline Diabetes mellitus & $19(31.7)$ & $24(32.4)$ & $17(35 \cdot 4)$ & 0.571 \\
\hline Chronic liver disease & $5(6.7)$ & $5(6.8)$ & $5(10.4)$ & 0.655 \\
\hline Chronic kidney disease & $1(1.3)^{\mathrm{a}}$ & $12(16.5)$ & $9(18.8)^{b}$ & 0.002 \\
\hline Cerebrovascular disease & $23(30.7)^{c}$ & $41(55 \cdot 4)$ & $21(43.8)$ & 0.009 \\
\hline Rheumatoid disease & $1(1.3)$ & $2(2.7)$ & 0 & 0.485 \\
\hline Current malignancy & $7(9 \cdot 3)$ & $24(32.4)$ & $17(37.0)$ & 0.095 \\
\hline \multicolumn{5}{|l|}{ Radiographic finding } \\
\hline Bilateral lung involvement & $54(72.0)$ & $51(71.8)$ & $27(56.3)$ & 0.130 \\
\hline Pleural effusion & $16(21.3)$ & $18(25.0)$ & $18(37.5)$ & 0.130 \\
\hline \multicolumn{5}{|l|}{ Clinical parameters } \\
\hline Leukopenia & $4(5 \cdot 3)$ & $17(23.0)$ & $5(10.6)$ & 0.005 \\
\hline C-reactive protein, mg/dL & $16.3 \pm 9.6$ & $15.6 \pm 9.7$ & $14.1 \pm 8.1$ & 0.440 \\
\hline Procalcitonin, ng/mL & $12.5 \pm 24.1$ & $13.7 \pm 28.0$ & $33 \cdot 5 \pm 57 \cdot 0$ & 0.080 \\
\hline ARDS & $19(25 \cdot 7)$ & $11(14 \cdot 9)$ & $12(25.0)$ & 0.229 \\
\hline Sepsis & $58(77 \cdot 3)$ & $66(89.2)$ & $42(91.3)$ & 0.800 \\
\hline Mechanical ventilation & $66(88.0)$ & $70(94.3)$ & $48(100)$ & 0.613 \\
\hline CRRT & $15(20.0)$ & $18(24 \cdot 3)$ & $14(29.2)$ & 0.132 \\
\hline \multicolumn{5}{|l|}{ Severity } \\
\hline APACHE II & $25.1 \pm 8.2$ & $27.1 \pm 10.4$ & $24.0 \pm 8.0$ & 0.152 \\
\hline SOFA (day 1 ) & $8.8 \pm 4.0$ & $9.89 \pm 4.3$ & $8.6 \pm 4.1$ & 0.153 \\
\hline PSI risk class $\geq \mathrm{IV}$ & $67(89.3)$ & $71(95.9)$ & $45(97.8)$ & 0.108 \\
\hline CURB- $65 \geq 3$ & $35(49.0)$ & $41(58.5)$ & $24(50)$ & 0.691 \\
\hline
\end{tabular}

Values are presented as median (range), number (\%), or mean $\pm \mathrm{SD}$.

CAP, community-acquired pneumonia; HCAP, healthcare-acquired pneumonia; HAP, hospital-acquired pneumonia; ARDS, acute respiratory distress syndrome; CRRT, continuous renal replacement therapy; APACHE II, Acute Physiology and Chronic Health Evaluation II; SOFA, Sequential Organ Failure Assessment; PSI, pneumonia severity index; CURB-65, confusion, urea, respiratory rate, age $\geq 65$.

${ }^{a} p<0.05$ when compared with HCAP.

${ }^{\mathrm{b}} \mathrm{p}<0.05$ when compared with CAP.

${ }^{\mathrm{c}}$ Chronic lung disease includes chronic obstructive lung disease and structural lung diseases, such as bronchiectasis.

Among the combination therapies, antipseudomonal $\beta$-lactamase in combination with fluoroquinolone was the most frequently used in HCAP and HAP $39.2 \%$ and $34.8 \%)$. $\beta$-Lactamase in combination with fluoroquinolone was the most common in CAP (34.6\%). Among the monotherapies, antipseudomonal $\beta$-lactamase was the most frequently used in three groups (CAP 6.7\%, HCAP
12.2\%, and HAP 17.4\%). Broad-spectrum antibiotics were administered to the CAP patients significantly less often than to the HCAP and HAP patients $(p<0.05)$.

There were also no significant differences in clinical outcomes, including ICU mortality, 28-day mortality, length of ICU stay, and the duration of mechanical ventilation (Table 5). The multiple logistic regression anal- 
Table 2. Distribution of HCAP $(\mathbf{n}=117)$

\begin{tabular}{lc}
\hline HCAP & Number \\
\hline $1^{\mathrm{a}}$ & 37 \\
$2^{\mathrm{b}}$ & 35 \\
$3^{\mathrm{c}}$ & 37 \\
$4^{\mathrm{d}}$ & 8 \\
\hline
\end{tabular}

The numbers add up to more than the total, as many patients presented more than one HCAP criteria.

HCAP, healthcare-acquired pneumonia.

${ }^{a}$ Hospitalization in an acute care hospital for 2 or more days within 90 days of the infection.

${ }^{\mathrm{b}}$ Infusion therapy, such as intravenous antibiotic therapy, chemotherapy, or wound care, within 30 days of a current infection.

${ }^{\mathrm{c}}$ Residence in a nursing home or long-term care facility.

${ }^{\mathrm{d}}$ Regular attendance at a dialysis clinic, including hemodialysis and peritoneal dialysis. ysis resulted in a significantly increased odds of mortality associated with the acute physiologic PSI score and treatment response (Table 6).

\section{DISCUSSION}

Previous studies have compared bacteriological differences and clinical outcomes between HCAP and CAP, or between HCAP and HAP [1,14-18]. A study compared HCAP with CAP and HAP at the same time without mention of ICU admission [19]. To our knowledge, this is the first report to compare the microbiologic epidemiology and clinical outcomes in patients admitted to the ICU with HCAP, to those with CAP and HAP. Three groups of pneumonia had similar baseline characteris-

Table 3. Distribution of the isolated pathogens in CAP, HCAP, and HAP patients

\begin{tabular}{|c|c|c|c|c|}
\hline Pathogen identified $^{\mathrm{a}}$ & $\operatorname{CAP}(\mathrm{n}=37)$ & $\operatorname{HCAP}(n=46)$ & $\operatorname{HAP}(\mathrm{n}=29)$ & $p$ value \\
\hline Gram-positive pathogen & $21(56.8)$ & $17(37.0)$ & $14(48.3)$ & 0.193 \\
\hline Streptococcus pneumoniae & $5(13.5)$ & $3(6.5)$ & o & 0.249 \\
\hline Streptococci other than S. pneumoniae & $3(8.1)$ & $2(4 \cdot 3)$ & o & 0.285 \\
\hline Staphylococcus aureus & $14(37.8)$ & $12(26,1)$ & $13(44.8)$ & 0.226 \\
\hline MSSA & $11(29.7)$ & $4(8.7)$ & o & 0.001 \\
\hline MRSA & $3(8.1)$ & $9(19.6)$ & $13(44.8)$ & 0.002 \\
\hline Gram-negative pathogen & $18(48.6)$ & $35(76.1)$ & $23(79 \cdot 3)$ & 0.009 \\
\hline Pseudomonas aeruginosa & $4(10.8)$ & $5(10.9)$ & $7(24.1)$ & 0.212 \\
\hline Klebsiella pneumoniae & $9(23 \cdot 3)$ & $21(45 \cdot 6)$ & $7(24 \cdot 1)$ & 0.060 \\
\hline Escherichia coli & $2(5 \cdot 4)$ & $7(15 \cdot 3)$ & $4(13.7)$ & 0.349 \\
\hline Enterobacter spp. & $2(5 \cdot 4)$ & $3(6.5)$ & $3(10.3)$ & 0.725 \\
\hline MDR & $5(13 \cdot 5)$ & $18(39.1)$ & $23(79 \cdot 3)$ & $<0.001$ \\
\hline MRSA & $3(8.1)$ & $9(19.6)$ & $13(44.8)$ & 0.002 \\
\hline ESBL producing Enterobacteriae ${ }^{\mathrm{b}}$ & $1(2.7)$ & $10(21.7)$ & $8(27.6)$ & 0.015 \\
\hline MDR-Pseudomonas spp. ${ }^{\mathrm{c}}$ & 0 & $1(2.2)$ & $2(6.9)$ & 0.221 \\
\hline CRAB & $1(2.7)$ & $3(6.5)$ & $4(13.8)$ & 0.219 \\
\hline Stenotrophomonas maltophilia & $1(2.7)$ & 0 & $2(6.9)$ & 0.200 \\
\hline Inappropriate antibiotics treatment & $4(11.8)$ & $15(32.6)^{\mathrm{d}}$ & $14(51.7)^{\mathrm{d}}$ & 0.006 \\
\hline
\end{tabular}

Values are presented as number (\%; no/patients with pathogen identified).

CAP, community-acquired pneumonia; HCAP, healthcare-acquired pneumonia; HAP, hospital-acquired pneumonia; MSSA, methicillin-susceptible Staphylococcus aureus; MRSA, methicillin-resistant Staphylococcus aureus; MDR, multidrug-resistant; ESBL, extended-spectrum $\beta$-lactamase; CRAB, carbapenem-resistant Acinetobacter baumannii.

${ }^{a}$ Numbers include mixed population of pathogens (4 in CAP, 7 in HCAP, and 9 in HAP).

${ }^{\mathrm{b}}$ ESBL producing Enterobacteriae include Klebsiella pneumoniae, Escherichia coli, Enterobacter spp.

${ }^{\mathrm{c}} \mathrm{MDR}$ Pseudomonas spp. means resistant Pseudomonas aeruginosa.

$\mathrm{d}_{\mathrm{p}}<0.05$ when compared with CAP. 


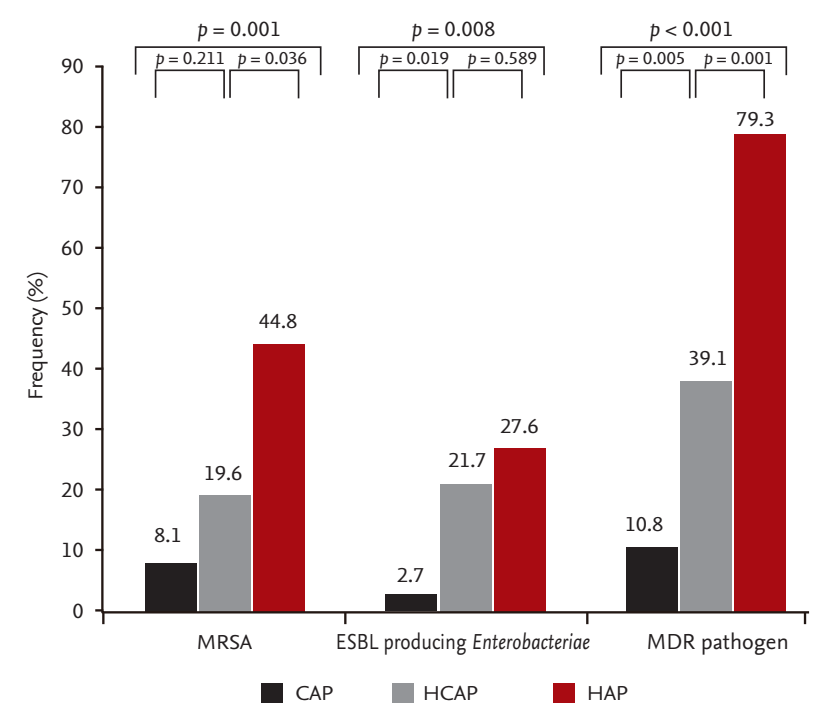

Figure 1. The distribution of multidrug-resistant (MDR) pneumonia pathogens in patients admitted to the intensive care unit for pneumonia, compared between three groups. CAP, community-acquired pneumonia; HCAP, healthcare-acquired pneumonia; HAP, hospital-acquired pneumonia; MRSA, methicillin-resistant Staphylococcus aureus; ESBL, extended-spectrum $\beta$-lactamase.

tics and pneumonia severity.

We identified the rate of MDR pathogens in the patients with HCAP was less than that in the patients with HAP and greater than that in patients with CAP as per the IDSA/ATS guidelines. However, the distribution of pathogens in the patients with HCAP was different from previous studies. Most common pathogen in HCAP reported previous studies was $S$. aureus or S. pneumonia $[1,9,14,20]$. In our study, K. pneumoniae (45.6\%) was the most common pathogen. Consequently, ESBL-producing $\mathrm{K}$. pneumoniae was also the most common MDR pathogen. The incidence of MRSA in the HCAP group (19.6\%) was similar to that in the CAP group $(8.1 \%, p=$ $0.221)$ and lower than that in the HAP group $(44.8 \%, p=$ 0.036). Similarly, one other study of microbial characteristics of HCAP and HAP in Korea showed similar microbial distribution. K. pneumoniae was the most common pathogen in HCAP group. The incidence of MRSA was lower than that of HAP group [21]. The explanation for these differences is not clear. A study in residents of long-term care facilities reported that the most common pneumonia pathogens were gram-negative bacilli
(18\%) [22]. Pop-Vicas and D'Agata [23] noted the factors that were independently associated with the isolation of MDR gram-negative bacilli in these patients were an age $>65$ years, prior antibiotic therapy for $>2$ weeks, and residence in a long-term care facility. These are similar to the definition for HCAP.

The rate of initial administration of broad-spectrum antibiotics in the patients with HCAP and HAP were higher than those in patients with CAP as per the IDSA/ATS guidelines [3]. Despite the more common use of broad spectrum antibiotics in the HCAP and HAP groups, the initial antibiotic treatment was inappropriate more frequently in the HCAP and HAP groups than in the CAP group. This difference may be explained by the differing prevalence of MDR pathogens between the groups. ESBL-producing K. pneumoniae was common in HCAP, whereas Pseudomonas spp. were less common in our study. According to our study, regional antimicrobial prescribing guidelines should contain the diversity in regional trends in microbial drug resistance.

Generally, the clinical course is poorer and the length of hospital stay is prolonged in patients with HCAP, compared to patients with CAP $[1,6,8]$. Our study failed to show a significant difference in the clinical outcomes among the three groups because of the disease severity who were requiring ICU care by itself. Our study population was characterized by high severity of disease, approaching PSI stage IV and V disease. Overall mortality at 28 days was more than $20 \%$ in all of three groups. In one previous study that reported poorer clinical outcomes in patients with HCAP than that in those with CAP for low-risk patients, the mortality rates were not different for the high-risk patients [24]. Especially, we did demonstrate that ICU mortality was associated with pneumonia severity. With similar disease severity, patients with CAP may demonstrate similar mortality as patients with HCAP or HAP, regardless of the presence of MDR pathogens.

Treatment response was another important factor for ICU mortality. Despite significant gradual differences among the groups in the rate of MDR pathogens and the presence of a high rate of broad-spectrum antibiotic use and inappropriate treatment in our study, there were no differences in the clinical outcomes including hospital length and mortality. Physician choice the initial antibiotics considering the risk factor of MDR pathogen or 
Table 4. Initial antibiotic treatment

\begin{tabular}{|c|c|c|c|c|}
\hline Empiric antibiotic & $\mathrm{CAP}(\mathrm{n}=75)$ & $\operatorname{HCAP}(n=74)$ & $\operatorname{HAP}(n=46)$ & $p$ value \\
\hline Monotherapy & $10(13 \cdot 3)$ & $16(21.6)$ & $13(26.7)$ & 0.175 \\
\hline$\beta$-Lactamase & $4(5 \cdot 3)$ & $2(2.7)$ & o & 0.254 \\
\hline Antipseudomonal $\beta$-lactamase & $5(6.7)$ & $9(12.2)$ & $8(17 \cdot 4)$ & 0.187 \\
\hline Vancomycin & $1(1.3)$ & $1(1.4)$ & o & 0.733 \\
\hline Carbapenem & $1(1.3)$ & $4(5 \cdot 4)$ & $4(8.7)$ & 0.051 \\
\hline Combination therapy & $65(86.7)$ & $58(78.4)$ & $33(71.7)$ & 0.175 \\
\hline$\beta$-Lactamase + Quinolone ${ }^{a}$ & $26(34 \cdot 6)$ & $7(9 \cdot 5)$ & $1(2.2)$ & $<0.001$ \\
\hline$\beta$-Lactamase + Macrolide & $1(1.3)$ & o & o & 0.449 \\
\hline$\beta$-Lactamase + Clindamycin & $6(8.0)$ & $8(11.0)$ & $3(6.5)$ & 0.568 \\
\hline Antipseudomonal $\beta$-lactamase + Quinolone ${ }^{a}$ & $25(33 \cdot 3)$ & $29(39.2)$ & $16(34.8)$ & 0.747 \\
\hline Antipseudomonal $\beta$-lactamase + Vancomycin & o & $1(1.4)$ & $3(6.5)$ & 0.043 \\
\hline Carbapenem + Quinolone ${ }^{\mathrm{a}}$ & o & $1(1.4)$ & 0 & \\
\hline Vancomycin + Carbapenem & $3(4 \cdot 0)$ & $7(9 \cdot 5)$ & $8(17 \cdot 4)$ & 0.048 \\
\hline Antipseudomonal $\beta$-lactamase + Quinolone + Vancomycin & $1(1.3)$ & $4(5.4)$ & $2(4 \cdot 3)$ & 0.392 \\
\hline Others & $2(2.6)$ & $1(1.4)$ & $1(2.2)$ & \\
\hline Broad spectrum antibiotics ${ }^{b}$ & $32(42.7)$ & $47(63.5)^{c}$ & $33(71.7)^{c}$ & 0.003 \\
\hline Treatment failure $^{\mathrm{d}}$ & $15(20.0)$ & $26(35 \cdot 1)$ & $19(41.3)$ & 0.076 \\
\hline
\end{tabular}

Values are presented as number (\%).

CAP, community-acquired pneumonia; HCAP, healthcare-acquired pneumonia; HAP, hospital-acquired pneumonia.

${ }^{\mathrm{a}} \mathrm{Quinolone}$ was levofloxacin.

${ }^{\mathrm{b}}$ Broad spectrum antibiotic use was defined as the use of any antibiotics including antipseudomonal $\beta$-lactamase or vancomycin or carbapenem.

${ }^{c} p<0.05$ when compared with CAP.

${ }^{\mathrm{d}}$ Treatment failure means death during initial treatment or change of empirical antibiotics from the initial agents to others on the 7 th day from medical intensive care unit admission.

Table 5. Clinical outcomes of study populations

\begin{tabular}{lcccc}
\hline Variable & CAP $(\mathrm{n}=75)$ & HCAP $(\mathrm{n}=74)$ & HAP $(\mathrm{n}=46)$ & $p$ value \\
\hline Duration, day & & & & \\
ICU & $10.48 \pm 11.9$ & $11.0 \pm 10.39$ & $12.65 \pm 11.20$ & 0.321 \\
MV & $10.15 \pm 12.54$ & $10.48 \pm 10.89$ & $12.44 \pm 11.29$ & 0.575 \\
Ventilator free days ${ }^{\mathrm{a}}$ & $2.0 \pm 1.90$ & $2.8 \pm 5.87$ & $2.06 \pm 2.69$ & 0.662 \\
ICU free days $^{\mathrm{b}}$ & $14.0 \pm 29.9$ & $27.9 \pm 29.0$ & $39.7 \pm 27.7$ & 0.831 \\
Mortality $_{\text {ICU mortality }}$ & $21(28.0)$ & $20(27.0)$ & & 0.124 \\
28-Day mortality & $26(38.2)$ & $22(32.8)$ & $14(31.1)$ & 0.694 \\
\hline
\end{tabular}

Values are presented as mean $\pm \mathrm{SD}$ or number $(\%)$.

CAP, community-acquired pneumonia; HCAP, healthcare-acquired pneumonia; HAP, hospital-acquired pneumonia; ICU, intensive care unit; MV, mechanical ventilation.

${ }^{a}$ A total of 92 patients were successfully weaned from mechanical ventilation in the ICU.

${ }^{\mathrm{b}} \mathrm{ICU}$ free days refers to the period from ICU discharge to hospital discharge. 
Table 6. Results of the logistic regression analysis to determine the factors associated with mortality

\begin{tabular}{lccc}
\hline Predictor & OR & $95 \%$ CI & $p$ value \\
\hline Male sex & 1.88 & $0.64-5.49$ & 0.249 \\
Age, yr & 1.03 & $0.99-1.08$ & 0.151 \\
CAP $^{\mathrm{a}}$ & 1.41 & $0.54-3.67$ & 0.477 \\
HAP & 1.80 & $0.63-5.15$ & 0.270 \\
PSI & 1.01 & $1.00-1.03$ & 0.036 \\
MDR pathogens & 0.45 & $0.15-1.10$ & 0.142 \\
Poor treatment response $^{\mathrm{b}}$ & 3.51 & $1.57-9.24$ & 0.003 \\
\hline
\end{tabular}

OR, odds ratio; CI, confidence interval; CAP, community-acquired pneumonia; HAP, hospital-acquired pneumonia; PSI, pneumonia severity index; MDR, multidrug-resistant.

${ }^{\mathrm{a}}$ Compared with healthcare-acquired pneumonia.

${ }^{\mathrm{b}}$ Change of empirical antibiotics from initial agents to others within the $7^{\text {th }}$ day.

disease severity at the time of admission. There were no definite criteria for evaluating treatment response under treatments. It is critical to identify patients at risk for non-response pneumonia using defined criteria to institute early appropriate therapy. El Solh et al. [17] evaluated treatment failure of severe pneumonia including nursing home residents. However, no specific definition of treatment failure was used. The parameters such as the PSI score, CURB-65, or APACHE II evaluate the severity of pneumonia at the time of admission and not response to treatment. We evaluated treatment response with definite criteria; a change in the empirical antibiotics from the initial agents within the 7 th day of the ICU admission. The appropriate stewardship of antibiotics considering the treatment response could be more important factor influencing better clinical outcomes in this population.

The present study analysed data retrospectively within a single institution, which is a limitation. However, data were collected from a prospective cohort of patients who required ICU admission, and uniform methods were used to detect pathogens. Sputum and blood samples were evaluated for all of the patients, and $>60 \%$ of the patients underwent a bronchoscopy to obtain specimens. Our successful pathogen identification rate of $57 \%(112 / 195)$ was high compared to the $20 \%$ to $50 \%$ reported in other prospectively designed studies $[1,4,23,24]$. Second, prior antibiotic use in the HCAP group could not be accurately estimated due to insufficient information in the medical records from other clinics. In Korea, there are a wide variety of long-term health care facili- ties including assisted-living, rehabilitation, haemodialysis, and convalescent hospital facilities where antibiotics could be administered. Therefore, the number of patients in the HCAP subgroup (Supplementary Table 1) that were identified by the receipt of intravenous antibiotic therapy within 30 days of a current infection could have been underestimated. Finally, we excluded subsequent pneumonia events from patients who experienced $\geq 2$ events in the same admission, potentially underestimating the number of HAP patients.

In conclusion, MDR pathogens were isolated in HCAP patients requiring ICU admission at intermediate rates between those of CAP and HAP. However, there were no significant differences among type of pneumonia in the clinical outcomes, including mortality.

\section{KEY MESSAGE}

1. Multidrug-resistant pathogens were isolated in healthcare-associated pneumonia patients requiring intensive care unit admission at intermediate rates between those of community-acquired pneumonia and hospital-acquired pneumonia.

2. There were no significant differences among type of pneumonia in the clinical outcomes, including mortality.

3. The mortality was associated with the acute physiologic pneumonia severity index score and treatment response. 


\section{Conflict of interest}

No potential conflict of interest relevant to this article was reported.

\section{REFERENCES}

1. Kollef MH, Shorr A, Tabak YP, Gupta V, Liu LZ, Johannes RS. Epidemiology and outcomes of health-care-associated pneumonia: results from a large US database of culture-positive pneumonia. Chest 2005;128:3854-3862.2.

2. Hiramatsu K, Niederman MS. Health-care-associated pneumonia: a new therapeutic paradigm. Chest 2005;128:3784-3787.

3. American Thoracic Society; Infectious Diseases Society of America. Guidelines for the management of adults with hospital-acquired, ventilator-associated, and healthcareassociated pneumonia. Am J Respir Crit Care Med 2005;171:388-416.

4. Shindo Y, Sato S, Maruyama E, et al. Health-care-associated pneumonia among hospitalized patients in a Japanese community hospital. Chest 2009;135:633-640.

5. Sopena N, Sabria M; Neunos 2000 Study Group. Multicenter study of hospital-acquired pneumonia in non-ICU patients. Chest 2005;127:213-219.

6. Abrahamian FM, Deblieux PM, Emerman CL, et al. Health care-associated pneumonia: identification and initial management in the ED. Am J Emerg Med 2008;26(6 Suppl):1-11.

7. Valles J, Calbo E, Anoro E, et al. Bloodstream infections in adults: importance of healthcare-associated infections. J Infect 2008;56:27-34.

8. Nseir S, Grailles G, Soury-Lavergne A, Minacori F, Alves I, Durocher A. Accuracy of American Thoracic Society/ Infectious Diseases Society of America criteria in predicting infection or colonization with multidrug-resistant bacteria at intensive-care unit admission. Clin Microbiol Infect 2010;16:902-908.

9. Garcia-Vidal C, Viasus D, Roset A, et al. Low incidence of multidrug-resistant organisms in patients with healthcare-associated pneumonia requiring hospitalization. Clin Microbiol Infect 2011;17:1659-1665.

10. Ewig S, Welte T, Chastre J, Torres A. Rethinking the concepts of community-acquired and health-care-associated pneumonia. Lancet Infect Dis 2010;10:279-287.

11. Brito V, Niederman MS. Healthcare-associated pneu- monia is a heterogeneous disease, and all patients do not need the same broad-spectrum antibiotic therapy as complex nosocomial pneumonia. Curr Opin Infect Dis 2009;22:316-325.

12. Webb BJ, Dangerfield BS, Pasha JS, Agrwal N, Vikram HR. Guideline-concordant antibiotic therapy and clinical outcomes in healthcare-associated pneumonia. Respir Med 2012;106:1606-1612.

13. Nseir S, Blazejewski C, Lubret R, Wallet F, Courcol R, Durocher A. Risk of acquiring multidrug-resistant Gramnegative bacilli from prior room occupants in the intensive care unit. Clin Microbiol Infect 2011;17:1201-1208.

14. Carratala J, Mykietiuk A, Fernandez-Sabe N, et al. Health care-associated pneumonia requiring hospital admission: epidemiology, antibiotic therapy, and clinical outcomes. Arch Intern Med 2007;167:1393-1399.

15. Yakovlev SV, Stratchounski LS, Woods GL, et al. Ertapenem versus cefepime for initial empirical treatment of pneumonia acquired in skilled-care facilities or in hospitals outside the intensive care unit. Eur J Clin Microbiol Infect Dis 2006;25:633-641.

16. Craven DE, Palladino R, McQuillen DP. Healthcare-associated pneumonia in adults: management principles to improve outcomes. Infect Dis Clin North Am 2004;18:939962.

17. El Solh AA, Pietrantoni C, Bhat A, Bhora M, Berbary E. Indicators of potentially drug-resistant bacteria in severe nursing home-acquired pneumonia. Clin Infect Dis 2004;39:474-480.

18. Depuydt P, Putman B, Benoit D, Buylaert W, De Paepe P. Nursing home residence is the main risk factor for increased mortality in healthcare-associated pneumonia. J Hosp Infect 2011;77:138-142.

19. Giannella M, Pinilla B, Capdevila JA, et al. Pneumonia treated in the internal medicine department: focus on healthcare-associated pneumonia. Clin Microbiol Infect 2012;18:786-794.

20. Ewig S, Klapdor B, Pletz MW, et al. Nursing-homeacquired pneumonia in Germany: an 8-year prospective multicentre study. Thorax 2012;67:132-138.

21. Yoon WK, Kim M, Kim YY, et al. The clinical and microbial characteristics of healthcare-associated pneumonia. Korean J Med 2010;78:709-716.

22. Muder RR. Pneumonia in residents of long-term care facilities: epidemiology, etiology, management, and prevention. Am J Med 1998;105:319-330. 
23. Pop-Vicas AE, D'Agata EM. The rising influx of multidrug-resistant gram-negative bacilli into a tertiary care hospital. Clin Infect Dis 2005;40:1792-1798.
24. Jung JY, Park MS, Kim YS, et al. Healthcare-associated pneumonia among hospitalized patients in a Korean tertiary hospital. BMC Infect Dis 2011;11:61. 
Supplementary Table 1. Subgroups of healthcare-acquired pneumonia

\begin{tabular}{|c|c|c|c|c|c|}
\hline Variable & $\begin{array}{l}\mathrm{HCAP} 1 \\
(\mathrm{n}=37)^{\mathrm{a}}\end{array}$ & $\begin{array}{l}\mathrm{HCAP}_{2} \\
(\mathrm{n}=35)^{\mathrm{b}}\end{array}$ & $\begin{array}{l}\mathrm{HCAP}_{3} \\
(\mathrm{n}=37)^{\mathrm{c}}\end{array}$ & $\begin{array}{l}\mathrm{HCAP}_{4} \\
(\mathrm{n}=8)^{\mathrm{d}}\end{array}$ & $\begin{array}{c}\text { Subgroup } \geq 2 \\
(\mathrm{n}=32)\end{array}$ \\
\hline APACHE II & $27.03 \pm 9.64$ & $27.06 \pm 11.42$ & $25.84 \pm 9.64$ & $28.25 \pm 11.67$ & $26.31 \pm 9.47$ \\
\hline SOFA & $9.16 \pm 3.93$ & $9.51 \pm 4.15$ & $9 \cdot 41 \pm 4 \cdot 37$ & $12.13 \pm 4.67$ & $9.47 \pm 4.24$ \\
\hline PSI risk class $\geq I V$ & $36(97 \cdot 3)$ & $34(47 \cdot 9)$ & $36(97 \cdot 3)$ & $8(100)$ & $32(100)$ \\
\hline CURB $-65 \geq 3$ & $18(48.6)$ & $16(45 \cdot 7)$ & $16(43.2)$ & $7(87.5)$ & $16(50)$ \\
\hline Gram positive pathogen & $10(27.0)$ & $4(11.4)$ & $11(29.7)$ & $1(12.5)$ & $7(21.9)$ \\
\hline Gram negative pathogen & $18(48.2)$ & $17(48.6)$ & $16(43.2)$ & $3(37.5)$ & $14(43.8)$ \\
\hline MDR & $9(24 \cdot 3)$ & $7(20.0)$ & $12(32.4)$ & $1(12.5)$ & $9(28.1)$ \\
\hline MRSA & $4(10.4)$ & $2(5 \cdot 7)$ & $6(16.2)$ & 0 & $3(9.4)$ \\
\hline ESBL producing Enterobacteriae & $6(16.2)$ & $4(11.4)$ & $5(13.5)$ & $1(12.5)$ & $5(15.6)$ \\
\hline Treatment failure & $12(32.4)$ & $11(31.4)$ & $13(35 \cdot 1)$ & $5(62.5)$ & $12(37 \cdot 5)$ \\
\hline \multicolumn{6}{|l|}{ Duration } \\
\hline $\mathrm{ICU}$ & 9.4 & $9 \cdot 3$ & 11.7 & $7 \cdot 6$ & 8.9 \\
\hline ICU free days ${ }^{f}$ & $17 \cdot 9$ & 18 & $15 \cdot 3$ & 25 & 17.8 \\
\hline \multicolumn{6}{|l|}{ Mortality } \\
\hline ICU & $9(24.3)$ & $10(28.6)$ & $4(10.8)$ & $3(37.5)$ & $7(21.9)$ \\
\hline Hospital & $18(48.6)$ & $15(42.9)$ & $9(24.3)$ & $4(50.0)$ & $13(40.6)$ \\
\hline
\end{tabular}

Values are presented as mean $\pm \mathrm{SD}$ or number $(\%)$.

HCAP, healthcare-acquired pneumonia; APACHE II, Acute Physiology and Chronic Health Evaluation II; SOFA, Sequential Organ Failure Assessment; PSI, pneumonia severity index; CURB-65, confusion, urea, respiratory rate, age $\geq 65$; MDR, multidrug-resistant; MRSA, methicillin-resistant Staphylococcus aureus; ESBL, extended-spectrum $\beta$-lactamase; ICU, intensive care unit.

${ }^{a}$ Hospitalization in an acute care hospital for 2 or more days within 90 days of the infection.

${ }^{b}$ Infusion therapy, such as intravenous antibiotic therapy, chemotherapy, or wound care, within 30 days of a current infection.

${ }^{c}$ Residence in a nursing home or long-term care facility.

${ }^{\mathrm{d}}$ Regular attendance at a dialysis clinic, including hemodialysis and peritoneal dialysis.

${ }^{\mathrm{e}}$ ESBL producing Enterobacteriae include Klebsiella pneumoniae, Escherichia coli, Enterobacter spp.

${ }^{\mathrm{f}} \mathrm{ICU}$ free days refers to the period from ICU discharge to hospital discharge. 
Supplementary Table 2. Subgroups of hospital-acquired pneumonia $(n=46)$

\begin{tabular}{|c|c|}
\hline Variable & No. (\%) \\
\hline Aspiration pneumonia & $21(15.6)$ \\
\hline Ventilator associated pneumonia & $9(19.6)$ \\
\hline Postoperative pneumonia & $4(8.7)$ \\
\hline Other pneumonia $^{a}$ & $12(26.0)$ \\
\hline
\end{tabular}

